doi:10.1016/j.atherosclerosis.2005.06.045

Copyright (c) 2005 Published by Elsevier Ltd.

\title{
Effects of rosuvastatin on postprandial leukocytes in mildly hyperlipidemic patients with premature coronary sclerosis
}

\author{
A.J.H.H.M. van Oostrom ${ }^{a}$, , H.W.M. Plokker ${ }^{b}$, B.S. van Asbeck ${ }^{a}$, T.J. \\ Rabelink $^{a}$, K.P.M. van Kessel ${ }^{a}$, E.H.J.M. Jansen ${ }^{c}$, C.D.A. Stehouwer ${ }^{d}$ and M. \\ Castro Cabezas ${ }^{\mathrm{a}, \mathrm{e}, *}$
}

\author{
${ }^{a}$ Department of Internal Medicine, University Medical Center Utrecht, The \\ Netherlands \\ bHeart Lung Institute Utrecht, The Netherlands \\ 'Laboratory for Health Effects Research, National Institute for Public Health and \\ Environment, Bilthoven, The Netherlands \\ ${ }^{\mathrm{d} D e p a r t m e n t}$ of Internal Medicine, University Hospital Maastricht, The Netherlands \\ ${ }^{\text {e}}$ St. Franciscus Gasthuis, Department of Internal Medicine, Endocrinology and \\ Vascular Medicine, P.O. Box 10900, 3004 BA Rotterdam, The Netherlands \\ * Corresponding author. Tel.: +31 10 4617267; fax: +31 104612692.
}

\begin{abstract}
We investigated whether pro-inflammatory aspects of the postprandial phase can be modulated by rosuvastatin in premature coronary artery disease (CAD) patients. Herefore standardized $8 \mathrm{~h}$ oral fat loading tests were performed offtreatment and after rosuvastatin $40 \mathrm{mg} / \mathrm{d}$ in 20 male CAD patients (50 \pm 4 years). The expression of leukocyte activation markers CD11a, CD11b, CD62L and CD66b was studied using flowcytometry. Migration of isolated neutrophils towards chemoattractants was determined in a fluorescence-based assay. Rosuvastatin did not affect baseline leukocyte counts nor the postprandial neutrophil increment (maximum mean increase $+10 \%$ pre- and $+14 \%$ posttreatment, $P<0.01$ for each). Rosuvastatin reduced baseline platelets (from $266 \pm 78$ to $225 \pm 74 \times 10^{9}$ cells $\left./ \mathrm{L}, P<0.001\right)$ and blunted the postprandial platelet count change (maximum mean increase $+6 \%, P=0.01$, and $0 \%$, respectively). The baseline expression of CD11a, CD11b and CD62L increased on most types of leukocytes by rosuvastatin, whereas the postprandial responses were unaffected. Pretreatment, postprandial neutrophil migration increased dosedependently, but there were no postprandial changes after rosuvastatin. The latter effect was unrelated to changes in lipoprotein concentrations.

In conclusion, in CAD patients postprandial pro-inflammatory and pro-coagulant changes can be modified by rosuvastatin. These apparently lipid-lowering independent effects may render protection against atherosclerosis.
\end{abstract}

\section{Introduction}

Leukocytes are a prerequisite for the development of atherosclerosis [1]. Preventing adherence of leukocytes to the endothelium results in improved endothelial function [2] and inhibition of plaque formation in animal models [3]. Obligatory for this adherence is a cytokine-controlled sequential upregulation of selectins and adhesion molecules on activated leukocytes and endothelial cells [4]. 
Large epidemiological studies have linked the leukocyte count to coronary artery disease (CAD) [5] and its risk factors [6]. From all leukocytes, neutrophils are best associated with CAD [5], which is unexpected since neutrophils are not present in the atherosclerotic lesion unless it is ruptured [7]. However, when activated neutrophils reside near the endothelium, the production of proinflammatory mediators, like cytokines, reactive oxygen species (ROS) and degradative enzymes, may harm the endothelium [8]. Activation of neutrophils and monocytes, as determined by the flowcytometric quantification of selectin and integrin expression, has been described in CAD and hypercholesterolemic patients [9].

In vitro and ex vivo, triglyceride (TG)-rich lipoproteins activate neutrophils and monocytes [10] and [11]. In healthy subjects, TG-related postprandial recruitment and activation of neutrophils and monocytes have been described [12] and [13], suggesting a postprandial pro-inflammatory situation and increased adhesive capacity of these cells [12]. Furthermore, these changes paralleled the production of pro-inflammatory cytokines and oxidative stress and may contribute to endothelial dysfunction [12] and [13]. Postprandial inflammatory changes may play a role in the atherosclerotic process and are important since people are postprandial most part of the day [14].

Statins (3-hydroxy-3-methyl-CoA reductase inhibitors) are potent drugs in the primary and secondary prevention of CAD [15] and have shown to reduce fasting and postprandial lipemia [14] and [16]. In some trials, the beneficial effects of statins were observed across the whole range of pre-treatment cholesterol levels suggesting that these effects may go beyond LDL-cholesterol reduction [17]. It is thought that the non-lipid-lowering effects involve improvement of endothelial function and anti-inflammatory, antithrombotic and antiproliferative effects [18]. Recently, a novel statin, rosuvastatin, has been introduced. Rosuvastatin has shown to reduce both cholesterol and fasting plasma TG concentrations [19] and may have anti-inflammatory effects on endothelial cells [20]. However, no data about effects on postprandial inflammatory changes are available, neither for rosuvastatin nor for any other statin.

In the present study we investigated postprandial inflammatory changes in premature CAD patients with mild fasting combined hyperlipidemia before and after rosuvastatin.

\section{Materials and methods}

\subsection{Subjects}

The study was approved by the Independent Ethics Committee of the Institutional Review Board of the University Medical Center Utrecht and the St. Antonius Hospital Nieuwegein. Males aged 40-55 years with angiographically established $C A D$, without any CAD event $<6$ months prescreening were included. Exclusion criteria were: diabetes mellitus, renal and/or liver disease, apolipoprotein E2/E2 genotype, body mass index $>30 \mathrm{~kg} / \mathrm{m}^{2}$, smoking and use of alcohol $>3 \mathrm{U} / \mathrm{d}$, antioxidant vitamins and anti-inflammatory drugs except from low-dose aspirin. Fasting plasma lipids, after washout of lipid-lowering medication for 4 weeks, had to be cholesterol $>5 \mathrm{mM}$ and plasma TG $>1.7 \mathrm{mM}$. 


\subsection{Study design}

Patients visited the hospital four times after an overnight fast ( $>12 \mathrm{~h}$ ) without drinking alcohol on the day before. The first visit consisted of anthropometric measurements and blood sampling. The second visit comprised the first oral fat loading test (OFLT), followed by rosuvastatin $20 \mathrm{mg} / \mathrm{d}$ for 1 month. Hereafter, patients visited the outpatient clinic for pill counting and control of plasma lipids and safety parameters. Subsequently, the patients started with rosuvastatin $40 \mathrm{mg} / \mathrm{d}$ for 1 month, followed by a second OFLT.

Before the OFLT, a cannula was placed for venous blood sampling followed by 30 minutes of rest. Fresh cream at a dose of $50 \mathrm{~g}$ fat and $3.75 \mathrm{~g}$ glucose $/ \mathrm{m}^{2}$ body surface was used as fat source [15]. The participants remained supine during each test and were only allowed to drink mineral water. Blood samples were obtained in sodium EDTA ( $2 \mathrm{mg} / \mathrm{mL}$ ) and sodium heparin (170 IU) at regular time intervals up to $8 \mathrm{~h}$. Samples for flowcytometry were stored at $4{ }^{\circ} \mathrm{C}$, those for leukocyte count, differentiation and isolation were kept at room temperature for $<30$ minutes. Other samples were chilled and centrifuged immediately; plasma was stored at $-80^{\circ} \mathrm{C}$.

\subsection{Analytical methods}

Blood cell counts and differentials were determined automatically (Celldyn- $3500^{\circledR}$, Abbott, Abbott Park, USA). ROS production was estimated by an ex vivo protein oxidation test (SHp, Diacron, Grosseto, Italy). This test is based on the reaction of 5,5-dithiobis-2-nitrobenzoic acid with free sulfhydryl groups in serum proteins resulting in a colored complex. Lower signal represents reduced free sulfhydryl groups and increased disulfide bonds in serum proteins, indicating increased in vivo oxidative stress. Interleukin-8 (IL-8) and soluble vascular cell adhesion molecule-1 (sVCAM-1) were measured by ELISA (Quantiglo, R\&D systems, Minneapolis, USA and Bender MedSystems, Vienna, Austria). HDL-cholesterol obtained after precipitation with Phosphotungstate/ $\mathrm{MgCl}_{2}$, and cholesterol and TG were measured by colorimetry (CHOD-PAP and GPO-PAP, respectively; Roche diagnostics, Germany). LDL-cholesterol was calculated by Friedewald formula. Apolipoprotein B was measured by nephelometry using monoclonal antibodies (Behring Diagnostics, Germany). Apolipoprotein E genotype was determined as described [12]. Glucose, creatinin, alanine aminotransferase, aspartate aminotransferase and creatinin kinase were measured by dry chemistry colorimetry (Vitros 250; Johnson \& Johnson, Rochester, USA). Insulin sensitivity was estimated by the HOMA index (homeostasis model assessment $=$ glucose $\times$ insulin/22.5).

\subsection{Leukocyte activation markers}

Using fluorescent monoclonal antibodies, surface expression of leukocyte activation markers was detected by direct immunofluorescence in duplo and evaluated by flowcytometry using a fluorescence activated cell counter (FACScan, Becton-Dickinson, Heidelberg, Germany) and Cell-Quest software (BectonDickinson) as described [12] and [13]. Two pairs of markers were studied: fluorescein isothiocynate (FITC) conjugated CD66b (CLB, Amsterdam, The Netherlands) and phycoerythrin (PE) conjugated CD11b (DAKO, Glostrup, Denmark) and a combination of FITC conjugated CD62L (Cymbus Biotechnology, Hants, UK) and PE conjugated CD11a (Diaclone, Besancon, France). CD11a, $\mathrm{CD} 11 \mathrm{~b}$ and $\mathrm{CD} 62 \mathrm{~L}$ are the most important markers for early adhesion to the endothelium [21]. CD66b is a neutrophil degranulation marker that is not expressed on lymphocytes and monocytes [22]. Neutrophils, lymphocytes and 
monocytes were identified by their characteristic forward and side scattering properties. The average of the duplo of the mean fluorescence intensity (MFI) per cell was given in arbitrary units. In each subject the series of postprandial measurements were performed with identical instrument settings.

\subsection{Neutrophil isolation}

Heparinized blood was diluted 1:1 with pyrogen-free PBS and layered onto a gradient of Histopaque (density 1.119; Sigma, St. Louis, USA) and Ficoll (Amersham Biosciences, Uppsala, Sweden), respectively. After 20 minutes centrifugation at $395 \times g$, neutrophils were collected from the Histopaque phase and subjected to hypotonic shock with water to lyse residual erythrocytes. Neutrophils were washed and suspended at $5 \times 10^{6}$ cells $/ \mathrm{mL}$ in RPMI 1640 (Gibco, Paisley, UK) containing $0.05 \%$ human serum albumin (HSA, CLB), providing on average a $>98 \%$ purified neutrophil suspension.

\subsection{Chemotaxis assay}

Ex vivo migration of isolated neutrophils towards chemoattractants was determined in a fluorescence-based assay, consisting of a 96-well microplate, sealed off with a $8 \mu \mathrm{m}$ pore polycarbonate filter (Neuro Probe Inc., Gaithersburg, USA) [23]. Lower wells contained $N$-formylmethionyl-leucyl-phenylalanine (fMLP, Sigma), recombinant IL-8 (PeproTech, Rocky Hill, USA) and recombinant complement factor $5 \mathrm{a}$ (C5a, Sigma) as chemoattractants. Upper wells, on top of the filters, contained $25 \mu \mathrm{l}$ of fluorescent labeled neutrophils. Therefore neutrophils in RPMI/HSA were labeled for 20 minutes at $21^{\circ} \mathrm{C}$ with $4 \mu \mathrm{M}$ calceinAM (Molecular probes, Eugene, USA). Cells were washed and suspended at $2.5 \times 10^{6}$ cells $/ \mathrm{mL}$ in Hanks' balanced salt solution (HBSS, Gibco) containing $1 \%$ HSA. Control bottom wells were filled with $25 \mu$ fluorescent labeled cells and served as maximum signal. After 45 minutes incubation at $37^{\circ} \mathrm{C}$ in a humidified $\mathrm{CO}_{2}$-incubator, the filters were rinsed with PBS and fluorescence was measured using an excitation and emission of 485 and $530 \mathrm{~nm}$, respectively. The percentage of migrated neutrophils was determined as fluorescence divided by the maximum signal. Specific migration was calculated after subtraction of spontaneous migration towards buffer containing wells. All assays were performed by the same investigator unaware of the type of samples tested.

\subsection{Statistics}

Data are expressed as mean \pm S.D. in the text and tables and as mean \pm S.E.M. in the figures. Baseline values and postprandial between-test differences, estimated as mean incremental integrated area under the curve (dAUC, calculated by subtracting the baseline value from each following measurement and using the trapezoidal rule, GraphPad Prism version 3.0) were tested by a paired $t$-test. Time effects compared with $T=0 \mathrm{~h}$, were tested using repeated measures (RM)-ANOVA with least significant difference test. TG and leukocyte calculations were performed after logarithmic transformation. Bivariate correlations were calculated using Pearson's correlation coefficients. For statistical analysis SPSS version 10.0 was used. $P$-values $<0.05$ (2-tailed) were considered statistically significant. 


\section{Results}

\subsection{Subject characteristics and effects of rosuvastatin on lipemia}

From 22 screened patients, one was excluded due to the presence of the apolipoprotein E2/E2 genotype and one due to current smoking. At baseline patients showed a mild fasting combined hyperlipidemia (Table 1). Rosuvastatin $40 \mathrm{mg} /$ day was well tolerated and improved significantly fasting TG, LDLcholesterol, apolipoprotein B and HDL-cholesterol. TG-AUC was significantly reduced, whereas TG-dAUC was unaffected. Details on postprandial lipoprotein and fatty acid changes will be published separately. During treatment there were no significant changes in anthropometric variables and co-medication (low-dose aspirin $(n=19), \beta$-blockers $(n=13)$ and angiotensin converting enzyme inhibitors $(n=11))$.

Table 1.

Characteristics (mean (S.D.)) of 20 male CAD patients, off lipid lowering medication and after rosuvastatin $40 \mathrm{mg} / \mathrm{d}$

\begin{tabular}{|c|c|c|c|}
\hline & Pre-treatment & $40 \mathrm{mg} / \mathrm{d}$ rosuvastatin & Percentage change \\
\hline Age (year) & $50(4)$ & $50(4)$ & \\
\hline Body mass index $\left(\mathrm{kg} / \mathrm{m}^{2}\right)$ & $26.4(1.4)$ & $26.6(1.4)$ & \\
\hline Waist $(\mathrm{m})$ & $0.96(0.05)$ & $0.96(0.06)$ & \\
\hline Systolic blood pressure $(\mathrm{mmHg})$ & $129(12)$ & $130(14)$ & \\
\hline Diastolic blood pressure $(\mathrm{mmHg})$ & $87(8)$ & $88(9)$ & \\
\hline Glucose $(\mathrm{mM})$ & $5.2(0.5)$ & $5.5(0.6)$ & \\
\hline Insulin (mU/L) & $8.70(7.19)$ & ND & \\
\hline HOMA index & $2.04(1.72)$ & ND & \\
\hline Plasma triglycerides (mM) & $2.21(0.87)$ & $1.52(0.61)^{* *}$ & -31 \\
\hline TG-AUC (mmol h/L) & $28.5(9.2)$ & $22.0(7.1)^{* *}$ & -23 \\
\hline TG-dAUC (mmol h/L) & $6.43(4.21)$ & $6.83(3.66)$ & +6 \\
\hline Cholesterol (mM) & $6.3(1.0)$ & $3.6(0.6)^{* *}$ & -43 \\
\hline LDL-cholesterol (mM) & $4.4(1.0)$ & $1.8(0.5)^{* *}$ & -59 \\
\hline HDL-cholesterol (mM) & $0.93(0.23)$ & $1.10(0.32)^{*}$ & +18 \\
\hline Non HDL-cholesterol (mM) & $5.4(0.9)$ & $2.5(0.6)^{* *}$ & -54 \\
\hline Cholesterol/HDL-cholesterol & $7.1(1.7)$ & $3.5(0.9)^{* *}$ & -51 \\
\hline Apolipoprotein B $(\mathrm{g} / \mathrm{L})$ & $1.26(0.19)$ & $0.70(0.14)^{* *}$ & -44 \\
\hline Creatinin $(\mu \mathrm{M})$ & $91(12)$ & $91(12)$ & \\
\hline AST (U/L) & $32(10)$ & $35(12)$ & \\
\hline $\operatorname{ALT}(\mathrm{U} / \mathrm{L})$ & $34(23)$ & $40(18)$ & \\
\hline Creatinin kinase (U/L) & $130(91)$ & $159(117)$ & \\
\hline
\end{tabular}

HOMA, homeostasis model assessment; ND, not determined; TG-AUC, total area under the postprandial TG curve; TG-dAUC, incremental area under the curve.

${ }^{*} P<0.01$, paired t-test.

${ }^{* *} P<0.001$, paired $t$-test.

\subsection{Blood cell counts}

Rosuvastatin reduced significantly the baseline count of platelets and erythrocytes, but not of leukocytes (Table 2). These effects of treatment were not related significantly to rosuvastatin induced improvements of fasting and postprandial lipids (data not shown). Postprandial changes of cell counts (dAUC) were not significantly affected by treatment. Both postprandial tests showed a significant neutrophil count increment (Fig. 1A), a gradual lymphocyte increase 
(Fig. 1B) and monocyte counts without major changes except an initial decline (Fig. 1C). Pre-treatment there was a significant postprandial platelet increase to a maximum at $T=4 \mathrm{~h}$, that was not observed during the second test (Fig. 2A). Both postprandial tests showed a gradual significant decline in erythrocyte counts (Fig. 2B).

\section{Table 2.}

Fasting blood cell count and expression of activation markers on leukocytes (mean (S.D.)) in 20 male CAD patients, before and after rosuvastatin $40 \mathrm{mg} / \mathrm{d}$

\begin{tabular}{|l|l|l|}
\hline & Pre-treatment & $\mathbf{4 0 ~}$ mg/d rosuvastatin \\
\hline Erythrocytes $\left(10^{12}\right.$ cells/L) & $4.75(0.41)$ & $4.43(0.33)^{* *}$ \\
\hline Leukocytes $\left(10^{9}\right.$ cells/L) & $5.74(1.50)$ & $6.20(2.06)$ \\
\hline Neutrophils $\left(10^{9}\right.$ cells/L) & $3.13(1.22)$ & $3.46(1.52)$ \\
\hline Lymphocytes $\left(10^{9}\right.$ cells/L) & $1.80(0.36)$ & $1.94(0.56)$ \\
\hline Monocytes $\left(10^{9}\right.$ cells/L) & $0.54(0.18)$ & $0.53(0.15)$ \\
\hline Platelets $\left(10^{9}\right.$ cells/L) & $266(78)$ & $225(74)^{* *}$ \\
\hline \multicolumn{3}{|l|}{} \\
\hline CD11a monocytes (AU) & $60(6)$ & $73(13)^{* *}$ \\
\hline CD11a lymphocytes (AU) & $50(7)$ & $58(11)^{* *}$ \\
\hline CD11a neutrophils (AU) & $31(4)$ & $38(6)^{* *}$ \\
\hline CD11b monocytes (AU) & $158(31)$ & $186(36)^{* *}$ \\
\hline CD11b Iymphocytes (AU) & $33(9)$ & $36(11)^{*}$ \\
\hline CD11b neutrophils (AU) & $203(75)$ & $192(85)$ \\
\hline CD62L monocytes (AU) & $79(14)$ & $91(20)^{*}$ \\
\hline CD62L lymphocytes (AU) & $50(9)$ & $53(10)$ \\
\hline CD62L neutrophils (AU) & $80(14)$ & $88(17)$ \\
\hline CD66b neutrophils (AU) & $10.3(8.4)$ & $11.0(3.1)$ \\
\hline
\end{tabular}

Expression of CD markers on leukocytes was measured as mean fluorescence intensity (MFI) in arbitrary units (AU).

${ }^{*} P<0.01$, paired $t$-test.

${ }^{* *} P<0.001$, paired $t$-test. 

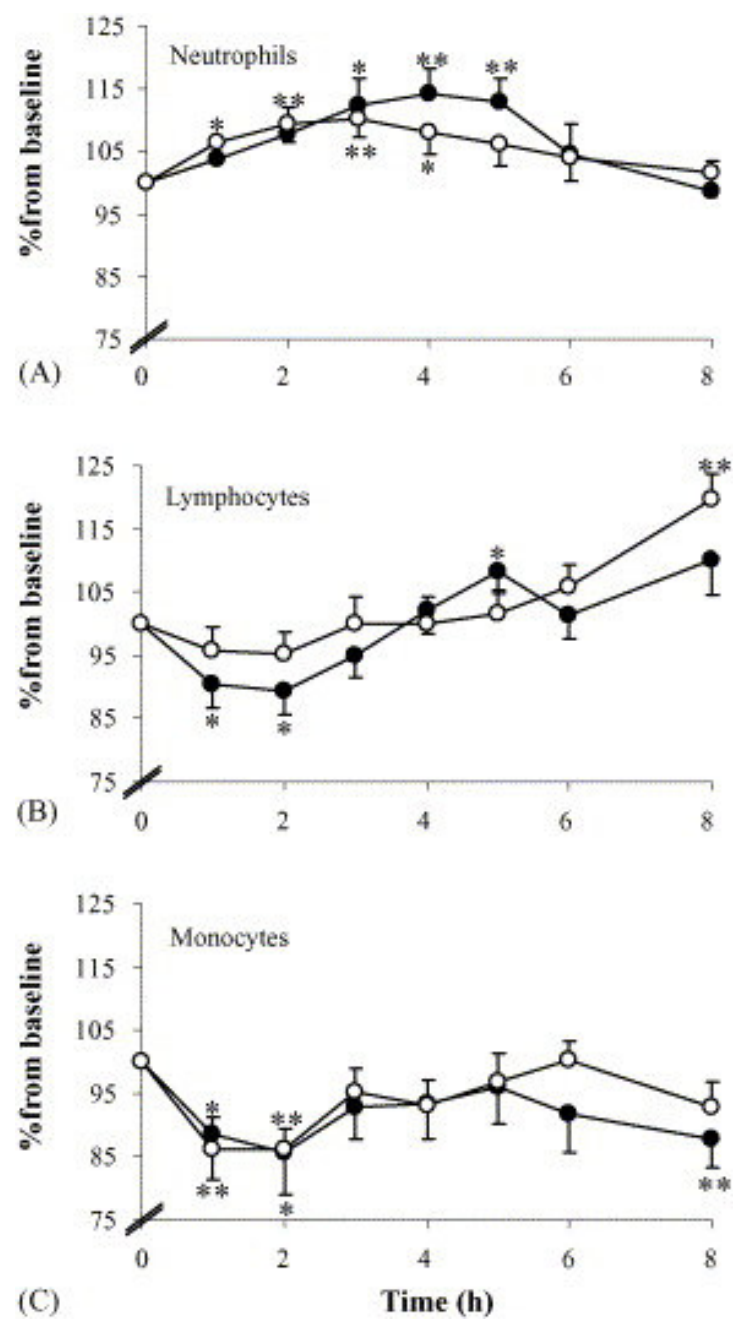

Fig. 1. Mean \pm S.E.M. relative postprandial changes of: neutrophil $(A)$; lymphocyte (B) and monocyte count (C) in 20 CAD patients before (closed bullets) and after rosuvastatin $40 \mathrm{mg} / \mathrm{d}$ (open bullets). Between test differences (paired $t$-test for incremental AUC's): $P=$ ns for all panels. Within test differences (RM-ANOVA): ${ }^{*} P<0.05,{ }^{* *} P<0.005$ vs. baseline. 

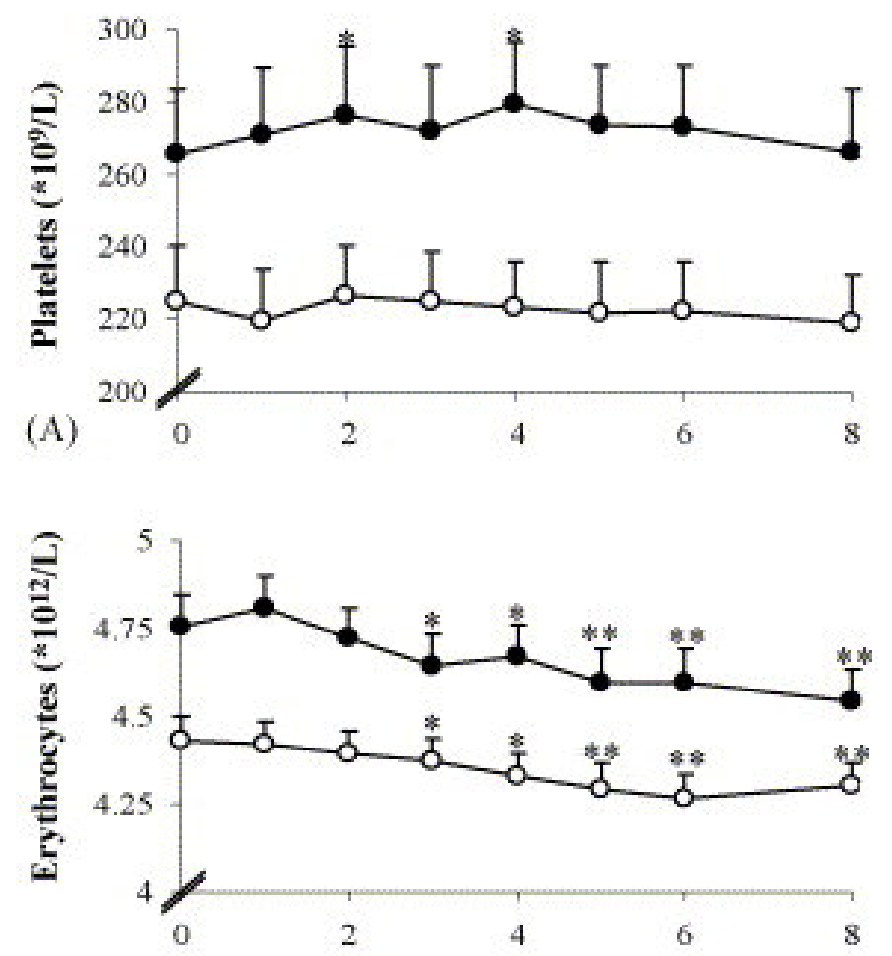

Time (h)

Fig. 2. Mean \pm S.E.M. postprandial platelet $(A)$ and erythrocyte count (B) in 20 CAD patients before (closed bullets) and after rosuvastatin $40 \mathrm{mg} / \mathrm{d}$ (open bullets). Between test differences (paired $t$-test for incremental AUC's): $P=$ ns for all panels. Within test differences (RM-ANOVA): ${ }^{*} P<0.05,{ }^{* *} P<0.005$ vs. baseline.

\subsection{Leukocyte activation markers}

Post-treatment, the fasting expression CD11a on all three investigated types of leukocytes, CD11b on monocytes and lymphocytes and of CD62L on monocytes, was significantly increased (Table 2). The increments in fasting CD11a on neutrophils and monocytes appeared related to the post-treatment reduction in fasting total cholesterol ( $R=0.48$ and $R=0.46$, respectively, $P<0.05$ for both). The rise in fasting neutrophil CD11a was also related to the reduction in fasting apoB $(R=0.62, P<0.005)$.

Treatment did not result in different postprandial expression of the investigated markers except for a small but significantly higher postprandial lymphocyte CD62L increase $(P=0.01$, Fig. 3). Postprandially in both tests, there were no significant changes in CD66b, whereas the other markers, except CD11b on lymphocytes, showed a significant gradual postprandial increase to a maximum at $T=8 \mathrm{~h}$. The largest increments were seen for neutrophil CD11b $(+50 \%)$. 

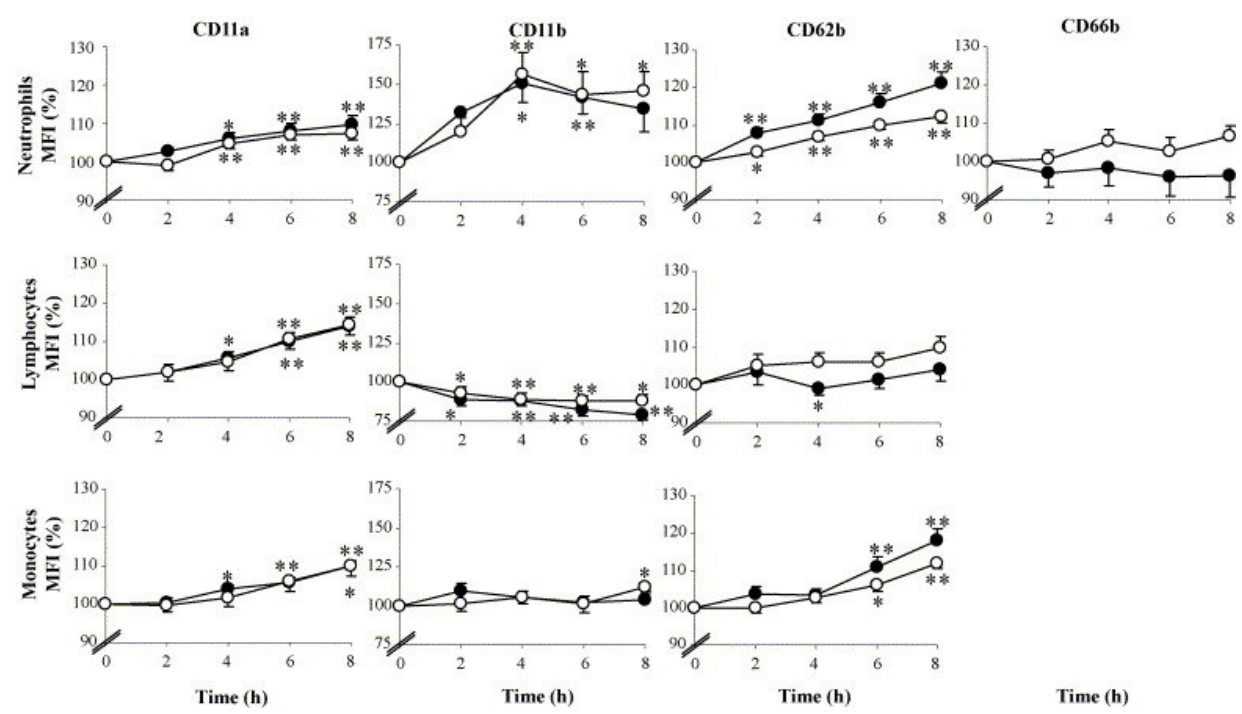

Time (h)

Fig. 3. Mean \pm S.E.M. relative postprandial changes in mean fluorescence intensity (MFI) of CD11a (first column), CD11b (second column), CD62L (third column) and CD66b (fourth column) on leukocytes in 20 CAD patients before (closed bullets) and after rosuvastatin $40 \mathrm{mg} / \mathrm{d}$ (open bullets). The upper, middle and lower panels represent neutrophils, lymphocytes and monocytes, respectively. Note the different $Y$-axis for CD11b. Between test differences (paired $t$-test for incremental AUC's): $P=0.01$ for CD62L on lymphocytes, $P=$ ns for the other panels. Within test differences (RM-ANOVA): ${ }^{*} P<0.05,{ }^{* *} P<0.005$ vs. baseline.

\subsection{Oxidative stress, sVCAM and interleukin-8,}

Post-treatment the fasting ex vivo protein oxidation capacity was increased (443 $\pm 29 \mu \mathrm{M}$ versus $425 \pm 33 \mu \mathrm{M}, P<0.005$, Fig. 4), suggestive of lower in vivo protein oxidation. Both postprandial tests showed a similar decline, suggesting a comparable postprandial increased protein oxidation.

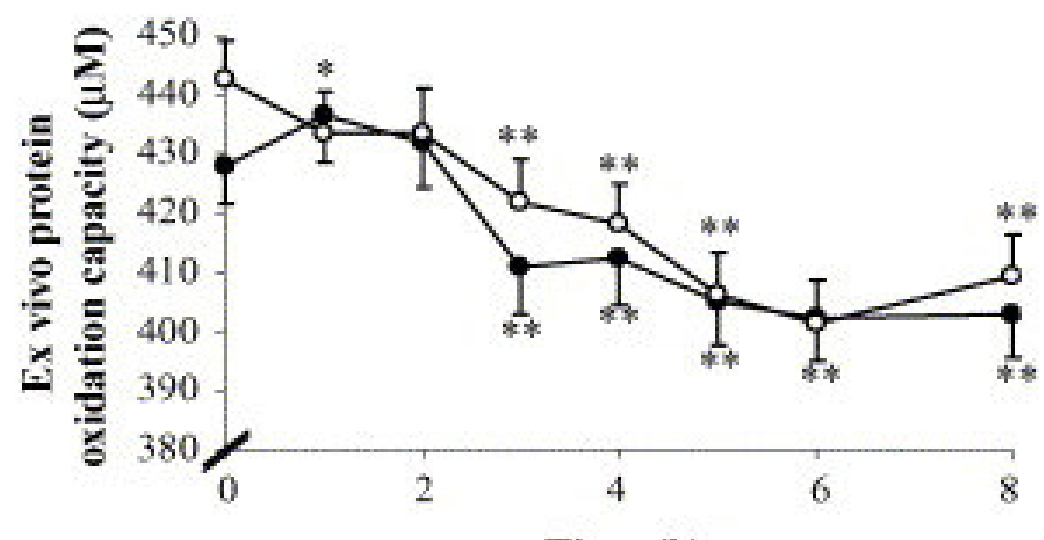

Time (h)

Fig. 4. Mean \pm S.E.M. postprandial ex vivo protein oxidation capacity in 20 CAD patients before (closed bullets) and after rosuvastatin $40 \mathrm{mg} / \mathrm{d}$ (open bullets). Between test differences (paired $t$-test for incremental AUC's): $P=n s$. Within test differences (RM-ANOVA): ${ }^{*} P<0.05,{ }^{* *} P<0.005$ vs. baseline. 
Upon treatment, fasting SVCAM-1 $(677 \pm 34 \mathrm{ng} / \mathrm{mL})$ was unaffected, postprandial changes were absent and not different from the pre-treatment test (data not shown).

Post-treatment, fasting IL-8 was significantly reduced (to $6.00 \pm 0.17 \mathrm{pg} / \mathrm{mL}$ ) whereas the postprandial response was increased. The latter was due to a gradual IL-8 increase to a maximum at $T=4 \mathrm{~h}(6.10 \pm 0.19 \mathrm{pg} / \mathrm{mL}, P<0.01)$ in contrast to an initial IL-8 decrease between $T=0-2 \mathrm{~h}$ (from $6.32 \pm 0.22 \mathrm{pg} / \mathrm{mL}$ to $6.17 \pm 0.10 \mathrm{pg} / \mathrm{mL}, P<0.005)$ pre-treatment. The effects of rosuvastatin on fasting oxidative capacity and IL-8 were unrelated to lipid changes (data not shown).

\subsection{Neutrophil migration}

Migration experiments were performed in eight patients. In both tests, migration of fasting and postprandial neutrophils towards C5a, IL-8 and fMLP occurred in a dose-dependent manner (Fig. 5). Pre-treatment, the concentration-migration responses of postprandial neutrophils shifted upward when compared to the response of fasting neutrophils, suggestive of meal-induced migration. When migration towards the lowest concentration chemoattractant was studied, e.g. the most sensitive range, the neutrophils at $T=8 \mathrm{~h}$ showed a 95,115 and $134 \%$ higher migration towards $\mathrm{C} 5 \mathrm{a}$, IL-8 and $\mathrm{FMLP}$, respectively, when compared to fasting neutrophils $(P<0.05$, Fig. 6A). Post-treatment, the upward shift of postprandial neutrophil migration was abolished and there were no marked differences in postprandial response at the lowest concentration of chemoattractant (Fig. 5 and Fig. 6B). The post-treatment reduction in migration was unrelated to improvement of lipoprotein concentrations or other treatmentinduced inflammatory changes (data not shown).
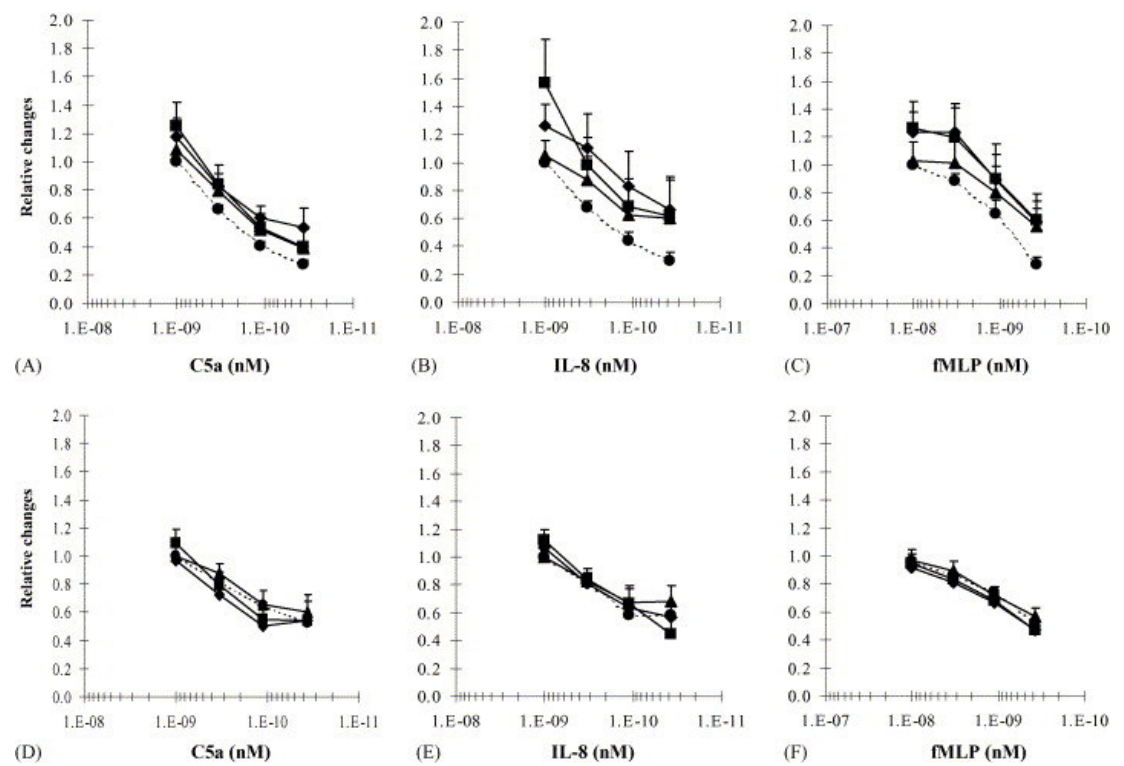

Fig. 5. Mean \pm S.E.M. neutrophil migration towards decreasing concentrations of complement component 5a (C5a), interleukin-8 (IL-8) and $\mathrm{N}$-formylmethionylleucyl-phenylalanine (fMLP) as chemoattractants in eight CAD patients. Data are shown for baseline (dotted line, closed bullets), $T=4$ (squares), $T=6$ (triangles) and $T=8$ (diamonds) postprandially, before treatment (panels a-c) and after rosuvastatin $40 \mathrm{mg} / \mathrm{d}$ (panels $\mathrm{d}-\mathrm{f}$ ). Data are expressed as relative changes in comparison to the highest concentration chemoattractant at baseline. 

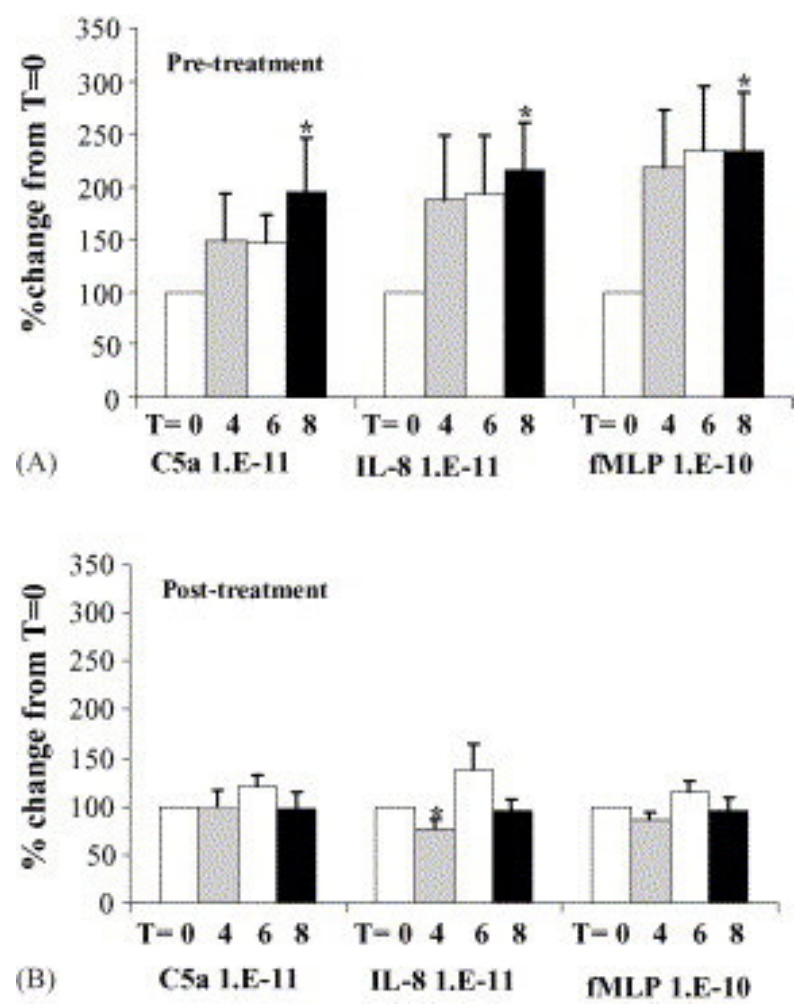

Fig. 6. Mean \pm S.E.M. neutrophil migration towards the lowest concentration of complement component 5a (C5a), interleukin-8 (IL-8) and $N$-formylmethionylleucyl-phenylalanine (fMLP) as chemoattractants in 20 CAD patients before (A) and after rosuvastatin $40 \mathrm{mg} / \mathrm{d}(B)$. Data are shown as relative change compared to baseline $(T=0)$. ${ }^{*} P<0.05$ vs. baseline (RM-ANOVA).

\section{Discussion}

This is the first study in CAD patients to investigate postprandial inflammatory changes and the effects of statin therapy on these processes. In general, rosuvastatin increased fasting parameters of inflammation, whereas postprandial changes were due to meal-feeding and unaffected by treatment except for a reduction of the postprandial migration capacity of neutrophils.

Based on previous findings, a reduced leukocyte count after rosuvastatin may have been expected [24]. However, in agreement with a different prospective statin-intervention study in hyperlipidemic patients [25] we did not find a change of fasting leukocyte count. Postprandially, we observed a neutrophil increment, unaffected by rosuvastatin. Previously, we have shown that neutrophil increments are meal-specific, in contrast to changes in lymphocyte and monocyte count that result from a diurnal rhythm [12].

Increased ex vivo adherence of monocytes and neutrophils to the endothelium from hyperlipidemic patients, and reduction of this adherence by statins has been shown earlier [9]. We have shown previously in healthy subjects and now in CAD patients, postprandial increments of leukocyte markers involved in adherence to the endothelium, in the former group these increments appeared to be caused by the ingestion of fat [13] and [21]. Rosuvastatin increased baseline CD11a and CD11b, which contrasts with studies showing no effect or reduced markers in 
hypercholesterolemic patients after atorvastatin, simvastatin and lovastatin [9], [25] and [26]. In theory, these rosuvastatin effects are not desirable, however, the exact in vivo relevance is not well known. By contrast, rosuvastatin increased baseline monocyte $\mathrm{CD} 62 \mathrm{~L}$, suggesting reduced activation since this marker is shed upon activation [13], similar effects have been reported for simvastatin but not for atorvastatin [25] and [26]. Postprandial marker increments were not affected by rosuvastatin, except for a higher lymphocyte CD62L increase, suggesting reduced activation.

Before treatment we observed increased postprandial chemotaxis of isolated neutrophils towards three different chemoattractants. Cell movement is a complex process, involving cytoskeleton alterations and rearrangement and upregulation of chemoattractant receptors on activated cells [27]. Upregulation of monocyte IL-8 receptors by incubation with oxidized LDL has been shown earlier [28], our data suggest similar properties of postprandial lipoproteins with neutrophils. Rosuvastatin treatment blunted the pre-treatment postprandial increment of migration towards IL-8, C5a and fMLP. It has been shown earlier that cerivastatin reduced migration of neutrophils towards IL-8 in vitro [29], as far as we know, the present report is the first showing this effect ex vivo. Rosuvastatin effects on neutrophil adhesion molecule expression in our opinion could not have influenced chemotaxis since the ligands for these markers are not available in the assay. The rosuvastatin-induced improvement of lipoprotein metabolism was unrelated to the effect on neutrophil migration, which may suggest lipid-lowering independent effects. In line with a simvastatin study in hypercholesterolemic patients [30], fasting but not postprandial plasma IL-8 was reduced by rosuvastatin. IL- 8 is mainly produced by activated monocytes, neutrophils and endothelial cells and is the major cytokine responsible for neutrophil activation and recruitment [31]. Apparently, in the present study reduction of IL-8 did not affect neutrophil activation and recruitment.

In agreement with the present study, postprandial leukocyte activation has also been reported after an acute glucose load [32]. In these studies, timedependency between the postprandial peak TG or glucose levels and inflammatory changes was weak or absent. The direct effect of TG on leukocytes may result from uptake of TG-rich particles as has been suggested by preliminary results [14]. Considering TG-rich lipoproteins as initiators of leukocyte recruitment and activation, it was remarkable that reduction of postprandial plasma TG by treatment, did not affect postprandial leukocyte count and activation, but only neutrophil migration. This suggests that the number of TGrich lipoproteins is less important or that other unknown mechanisms are involved. One possibility could be that the type of fatty acids in postprandial TGrich lipoproteins is more important than the particle number.

Lipoproteins can activate endothelial cells [33], which may indirectly lead to leukocyte activation as has been shown in angioplasty-induced endothelial cell activation [34]. As marker for endothelial cell activation we have measured sVCAM-1, which binds leukocytes via integrins such as CD11b and has been positively associated to CAD [35]. When compared with a postprandial study in type 2 diabetes patients [35], we observed a much smaller postprandial sVCAM-1 increment ( $30 \%$ versus $3 \%$ in our study) that was abolished by rosuvastatin. It is uncertain whether these increments reflect enhanced synthesis or in part result from increased shedding.

Previously we suggested that besides direct effects of lipoproteins, also a postprandial neutrophil increment may contribute to endothelial dysfunction via ROS or degradative enzyme production [12]. The role of leukocytes in endothelial 
dysfunction has also been described in ischemia-reperfusion-models [8] and is underlined by showing that endothelial dysfunction can be reversed when adherence of leukocytes is prevented [2]. In the present study ex vivo protein oxidizability was reduced postprandially, suggesting that oxidation occurred in vivo. Rosuvastatin did not affect postprandial oxidation, but reduced fasting oxidation. Unfortunately, we were not able to perform additional tests to assess other components of the oxidation status.

Leukocyte activation in theory should lead to adherence to the endothelium, resulting in underestimation of the in vivo situation by peripheral blood sampling. Indeed it has been shown that angioplasty-induced leukocyte activation is higher on locally obtained cells than on cells in peripheral blood [36]. Adherence most certainly is further enhanced due to endothelial cell activation by postprandial lipemia [33] or due to atherosclerotic disease in general. The lower endothelial cell activation after rosuvastatin may have reduced leukocyte adherence, which may in part explain the higher baseline expression of leukocyte activation markers post-treatment. Another argument for underestimation might be that the postprandial increment of activation markers and cell count was much lower than observed earlier in healthy controls [12] and [13].

In the present study we observed a postprandial platelet increase more or less comparable to healthy controls [13]. In that particular study we excluded stress and blood sampling effects. Increased postprandial platelet activation has been described previously [37]. This activation has possible prothrombotic effects and may induce activation of leukocytes, a mechanism that could have been operational in the present study [37] and [38]. Surprisingly, rosuvastatin reduced the baseline platelet count and blunted the postprandial increment. Statins most certainly have antithrombotic effects such as increased fibrinolysis and reduced platelet activation [18]. Further experiments are necessary to investigate the relationships between postprandial lipemia and platelet and leukocyte changes.

Although the use of comedication was unchanged along the study, aspirin and ACE inhibitors may have influenced the results. Aspirin at high doses $(\mathrm{g} / \mathrm{d})$ has anti-inflammatory properties such as reduction of leukocyte-endothelium adherence [39] while low dose (75-500 mg/d) had no such effect [40]. Our patients used a dose of $80-100 \mathrm{mg} / \mathrm{d}$ aspirin. Anti-inflammatory effects of ACE inhibitors or angiotensin II inhibitors have also been described [41], however, effects on postprandial inflammatory changes have not been reported.

In conclusion, in CAD patients the postprandial phase is a pro-inflammatory situation characterized by endothelial cell activation and increased numbers, activation and migration capacity of neutrophils. These aspects of the postprandial phase that are potentially harmful and may contribute to cardiovascular disease were not related to postprandial plasma TG changes. Migration of neutrophils and endothelial cell activation, but not activation and recruitment of leukocytes, is improved by rosuvastatin. The anti-inflammatory effects of rosuvastatin may be independent from lipid lowering. It is unclear whether other statins also have these effects.

\section{Acknowledgement}

This study was supported by an unrestricted educational grant from AstraZeneca. The authors are grateful to J.W.J.M. Cremers and P.K. Beekhof for their excellent technical assistance. Dr. C.G. Schalkwijk is greatly acknowledged for his constructive remarks. 


\section{References}

[1] A.J. Lusis, Atherosclerosis, Nature 407 (2000), pp. 233-241.

[2] T. Murohara, M. Buerke and A.M. Lefer, Polymorphonuclear leukocyte-induced vasocontraction and endothelial dysfunction. Role of selectins, Arterioscler Thromb 14 (1994), pp. 1509-1519.

[3] Y. Huo and K. Ley, Adhesion molecules and atherogenesis, Acta Physiol Scand 173 (2001), pp. $35-43$.

[4] R.A. Worthylake and K. Burridge, Leukocyte transendothelial migration: orchestrating the underlying molecular machinery, Curr Opin Cell Biol 13 (2001), pp. 569-577.

[5] G.D. Friedman, A.L. Klatsky and A.B. Siegelaub, The leukocyte count as a predictor of myocardial infarction, N Engl J Med 290 (1974), pp. 1275-1278.

[6] Z.S. Huang, K.L. Chien, C.Y. Yang, K.S. Tsai and C.H. Wang, Peripheral differential leukocyte counts in humans vary with hyperlipidemia, smoking, and body mass index, Lipids 36 (2001), pp. 237-245.

[7] T. Naruko, M. Ueda and K. Haze et al., Neutrophil infiltration of culprit lesions in acute coronary syndromes, Circulation 106 (2002), pp. 2894-2900.

[8] J.E. Jordan, Z.Q. Zhao and J. Vinten-Johansen, The role of neutrophils in myocardial ischemiareperfusion injury, Cardiovasc Res 43 (1999), pp. 860-878.

[9] C. Weber, W. Erl, K.S. Weber and P.C. Weber, HMG-CoA reductase inhibitors decrease CD11b expression and CD11b-dependent adhesion of monocytes to endothelium and reduce increased adhesiveness of monocytes isolated from patients with hypercholesterolemia, J Am Coll Cardiol 30 (1997), pp. 1212-1217.

[10] D. Tripathy, P. Mohanty and S. Dhindsa et al., Elevation of free Fatty acids induces inflammation and impairs vascular reactivity in healthy subjects, Diabetes 52 (2003), pp. 2882-2887.

[11] G.J. Wanten, T.B. Geijtenbeek and R.A. Raymakers et al., Medium-chain, triglyceride-containing lipid emulsions increase human neutrophil beta2 integrin expression, adhesion, and degranulation, J Parenter Enteral Nutr 24 (2000), pp. 228-233.

[12] A.J. van Oostrom, T.P. Sijmonsma and C. Verseyden et al., Postprandial recruitment of neutrophils may contribute to endothelial dysfunction, J Lipid Res 44 (2003), pp. 576-583.

[13] A.J. van Oostrom, T.J. Rabelink and C. Verseyden et al., Activation of leukocytes by postprandial lipemia in healthy volunteers, Atherosclerosis 177 (2004), pp. 175-182.

[14] A.J. van Oostrom, J.P.H. van Wijk and M. Castro Cabezas, Lipemia, inflammation and atherosclerosis: novel opportunities in the understanding and treatment of atherosclerosis, Drugs 64 (2004) (Suppl 2), pp. 19-41.

[15] M.R. Law, N.J. Wald and A.R. Rudnicka, Quantifying effect of statins on low density lipoprotein cholesterol, ischaemic heart disease, and stroke: systematic review and meta-analysis, BMJ 326 (2003), p. 1423.

[16] C.J. Halkes, H. van Dijk and P.P. de Jaegere et al., Postprandial increase of complement component 3 in normolipidemic patients with coronary artery disease: effects of expanded-dose simvastatin, Arterioscler Thromb Vasc Biol 21 (2001), pp. 1526-1530.

[17] S.M. Grundy, J.I. Cleeman and C.N. Merz et al., Implications of recent clinical trials for the National Cholesterol Education Program Adult Treatment Panel III Guidelines, J Am Coll Cardiol 44 (2004), pp. 720-732.

[18] W. Palinski and C. Napoli, Unraveling pleiotropic effects of statins on plaque rupture, Arterioscler Thromb Vasc Biol 22 (2002), pp. 1745-1750. 
[19] J.W. Blasetto, E.A. Stein, W.V. Brown, R. Chitra and A. Raza, Efficacy of rosuvastatin compared with other statins at selected starting doses in hypercholesterolemic patients and in special population groups, Am J Cardiol 91 (2003), pp. 3C-10C.

[20] T.J. Stalker, A.M. Lefer and R. Scalia, A new HMG-CoA reductase inhibitor, rosuvastatin, exerts anti-inflammatory effects on the microvascular endothelium: the role of mevalonic acid, $\mathrm{Br} \mathrm{J}$ Pharmacol 133 (2001), pp. 406-412.

[21] C. Weber, Novel mechanistic concepts for the control of leukocyte transmigration: specialization of integrins, chemokines, and junctional molecules, J Mol Med 81 (2003), pp. 4-19.

[22] T.P. Ducker and K.M. Skubitz, Subcellular localization of CD66, CD67, and NCA in human neutrophils, J Leukoc Biol 52 (1992), pp. 11-16.

[23] C.W. Frevert, V.A. Wong, R.B. Goodman, R. Goodwin and T.R. Martin, Rapid fluorescence-based measurement of neutrophil migration in vitro, J Immunol Methods 213 (1998), pp. 41-52.

[24] C. Bickel, H.J. Rupprecht and S. Blankenberg et al., Influence of HMG-CoA reductase inhibitors on markers of coagulation, systemic inflammation and soluble cell adhesion, Int J Cardiol 82 (2002), pp. 25-31.

[25] C.V. Serrano Jr., V.M. Yoshida and M.L. Venturinelli et al., Effect of simvastatin on monocyte adhesion molecule expression in patients with hypercholesterolemia, Atherosclerosis 157 (2001), pp. 505-512.

[26] T. Stulc, k.M. Vrabli, Z. Kasalova, R. Ceska and I. Marinov, Atorvastatin reduces expression of leukocyte adhesion molecules in patients with hypercholesterolemia, Atherosclerosis 166 (2003), pp. 197-198.

[27] C.A. Parent and P.N. Devreotes, A cell's sense of direction, Science 284 (1999), pp. 765-770.

[28] Z.B. Lei, Z. Zhang and Q. Jing et al., OxLDL upregulates CXCR2 expression in monocytes via scavenger receptors and activation of p38 mitogen-activated protein kinase, Cardiovasc Res 53 (2002), pp. 524-532.

[29] N.C. Kaneider, C.M. Reinisch, S. Dunzendorfer, C. Meierhofer, A. Djanani and C.J. Wiedermann, Induction of apoptosis and inhibition of migration of inflammatory and vascular wall cells by cerivastatin, Atherosclerosis 158 (2001), pp. 23-33.

[30] A. Rezaie-Majd, T. Maca and R.A. Bucek et al., Simvastatin reduces expression of cytokines interleukin-6, interleukin- 8, and monocyte chemoattractant protein-1 in circulating monocytes from hypercholesterolemic patients, Arterioscler Thromb Vasc Biol 22 (2002), pp. 1194-1199.

[31] M. Baggiolini, B. Dewald and B. Moser, Interleukin-8 and related chemotactic cytokines--CXC and CC chemokines, Adv Immunol 55 (1994), pp. 97-179.

[32] M.J. Sampson, I.R. Davies, J.C. Brown, K. Ivory and D.A. Hughes, Monocyte and neutrophil adhesion molecule expression during acute hyperglycemia and after antioxidant treatment in type 2 diabetes and control patients, Arterioscler Thromb Vasc Biol 22 (2002), pp. 1187-1193.

[33] H. Doi, K. Kugiyama and H. Oka et al., Remnant lipoproteins induce proatherothrombogenic molecules in endothelial cells through a redox-sensitive mechanism, Circulation 102 (2000), pp. 670676.

[34] T. Inoue, T. Uchida, I. Yaguchi, Y. Sakai, K. Takayanagi and S. Morooka, Stent-induced expression and activation of the leukocyte integrin Mac-1 is associated with neointimal thickening and restenosis, Circulation 107 (2003), pp. 1757-1763.

[35] F. Nappo, K. Esposito and M. Cioffi et al., Postprandial endothelial activation in healthy subjects and in type 2 diabetic patients: role of fat and carbohydrate meals, J Am Coll Cardiol 39 (2002), pp. 1145-1150.

[36] A. Buffon, L.M. Biasucci, G. Liuzzo, G. D'Onofrio, F. Crea and A. Maseri, Widespread coronary inflammation in unstable angina, N Engl J Med 347 (2002), pp. 5-12. 
Atherosclerosis Volume 185, Issue 2 , April 2006, Pages 331-339

[37] A. Broijersen, F. Karpe, A. Hamsten, A.H. Goodall and P. Hjemdahl, Alimentary lipemia enhances the membrane expression of platelet P-selectin without affecting other markers of platelet activation, Atherosclerosis 137 (1998), pp. 107-113.

[38] I. Ott, F.J. Neumann, M. Gawaz, M. Schmitt and A. Schomig, Increased neutrophil-platelet adhesion in patients with unstable angina, Circulation 94 (1996), pp. 1239-1246.

[39] M.H. Pillinger, C. Capodici and P. Rosenthal et al., Modes of action of aspirin-like drugs: salicylates inhibit erk activation and integrin-dependent neutrophil adhesion, Proc Natl Acad Sci USA 95 (1998), pp. 14540-14545.

[40] R.R. Azar, S. Klayme and M. Germanos et al., Effects of aspirin ( $325 \mathrm{mg} /$ day) on serum highsensitivity C-reactive protein, cytokines, and adhesion molecules in healthy volunteers, Am J Cardiol 92 (2003), pp. 236-239.

[41] P. Dandona, V. Kumar and A. Aljada et al., Angiotensin II receptor blocker valsartan suppresses reactive oxygen species generation in leukocytes, nuclear factor-kappa $B$, in mononuclear cells of normal subjects: evidence of an antiinflammatory action, J Clin Endocrinol Metab 88 (2003), pp. 4496-4501. 